\title{
ENDOVASCULAR APPROACH FOR AN INTRACRANIAL MYCOTIC ANEURYSM ASSOCIATED WITH INFECTIVE ENDOCARDITIS
}

\author{
Junichi Utoh, MD, Yoshimasa Miyauchi, MD, Hiraaki Goto, MD, Hiroyuki Obayashi, MD, and Tomomi Hirata, MD, \\ Kumamoto, Japan
}

One of the most serious complications of infective endocarditis is mycotic cerebrovascular aneurysm, which can cause a lethal subarachnoid hemorrhage (SAH). ${ }^{1}$ The treatment of mycotic aneurysms in hemodynamically unstable patients is controversial because solid guidelines based on large prospective studies are lacking. Patients show a wide variety in size, number, and location of these intracranial lesions, in addition to varying in basic cardiac function. We report a case of ruptured mycotic aneurysm caused by infective endocarditis in a patient whose cardiac function was too severely compromised to permit an open craniotomy.

A 42-year-old man was admitted with a history of general fatigue, dyspnea, fever, and night sweats for a week. He had a high body temperature of $38^{\circ} \mathrm{C}$ and cardiac murmurs indicative of aortic and mitral regurgitation. A chest radiograph demonstrated cardiomegaly and severe pulmonary edema. An echocardiogram revealed vegetation and severe regurgitation of both the aortic and mitral valves. A diagnosis of infective endocarditis was confirmed by the isolation of Streptococcus viridans in multiple arterial blood cultures. The patient was treated in the intensive care unit with high doses of antibiotics (ampicillin $10 \mathrm{gm} /$ day and tobramycin 120 $\mathrm{mg} /$ day), diuretics, and catecholamines to control bacteremia and acute heart failure. After 6 days of intensive care, the patient's body temperature dropped below $37^{\circ} \mathrm{C}$ and further blood cultures were negative for pathogens. The patient's pulmonary edema and cardiomegaly also were alleviated. The patient was moved to a general floor, and double valve replacement was scheduled for the following week.

Early the next morning, however, the patient became comatose and had a temperature of $38.6^{\circ} \mathrm{C}$, tachycardia, hypertension, and respiratory distress. Mechanical ventilation under endotracheal intubation was started. Computed tomography demonstrated a massive SAH. Subsequent cerebral angiography revealed a $5 \mathrm{~mm}$ aneurysm arising from the $\mathrm{P} 2$ portion of the right posterior cerebral artery (Fig. 1). The patient was transferred to the intensive care unit. Despite conservative therapy, including respiratory support and administration of diuretics and catecholamines, the patient's condition was considered to be too hemodynamically unstable to allow him to undergo general anesthesia and an emergency craniotomy for direct clipping of the aneurysm. Emergency double valve

From the First Department of Surgery, Kumamoto University School of Medicine, Kumamoto, Japan.

J THORAC CARDIOVASC SURG 1995;110:557-9

Copyright $(1995$ by Mosby-Year Book, Inc.

$0022-5223 / 95 \$ 3.00+0 \quad \mathbf{1 2 / 5 4 / 6 2 6 1 7}$ replacement with heparin for cardiopulmonary bypass was also considered potentially dangerous in light of the intracranial lesion. After discussion with neurosurgeons, an endovascular approach was selected to control the intracranial lesion by embolization of the aneurysm and parent artery. This endovascular procedure was performed on the day following the onset of SAH. A Tracker catheter (Target Therapeutics Inc., San Jose, Calif.) was inserted onto the involved vessel just proximal to the aneurysm. The parent artery was embolized with ethylene vinyl alcohol until blood flow was arrested. Angiography after the embolization revealed complete obliteration of the aneurysm (Fig. 2).

Medications including betamethasone, glycerol, and trapidil were administered for 2 weeks after the procedure to prevent cerebral edema and vasospasm. The patient's level of consciousness gradually improved, although left hemiparesis was observed. On the fifteenth day after the onset of SAH, selective cerebral angiography confirmed that the treated lesion could no longer be seen. The next day, double valve replacement was performed according to a standard technique with cardiopulmonary bypass, mild hypothermia, and potassium cardioplasia. The postoperative course was uneventful, and the patient was discharged from the hospital to another clinic to continue rehabilitation on postoperative day 20 .

Mycotic aneurysms of the cerebral arteries occur in $2 \%$ to $10 \%$ of patients with infective endocarditis. ${ }^{1-3}$ Rupture of mycotic aneurysm is associated with a grave prognosis and an $80 \%$ mortality rate. ${ }^{1}$ Although progressive heart failure and systemic embolism are well-established indications for valve replacement in patients with bacterial endocarditis, treatment of mycotic aneurysms is still controversial because of the wide variability in the conditions of these aneurysms, ruptured or unruptured, their numbers and locations, and the extent of associated cardiac failure. There is uncertainty regarding the operative priority in patients with both intracranial aneurysm and acute heart failure. If a craniotomy for direct clipping is performed first, perioperative management could be insufficient to control progressive heart failure. On the other hand, if valve replacement is selected first, cardiopulmonary bypass with systemic heparinization could cause lethal neurologic deficits. There are no guidelines for decision in cases of such critically ill patients as the one described here.

Frizzell and colleagues ${ }^{4}$ described an endovascular technique for embolizing a mycotic intracranial aneurysm in a patient with infective endocarditis without heart failure. If bacteremia and heart failure can be controlled by conservative means, several treatment options are available, including a direct approach, an endovascular 


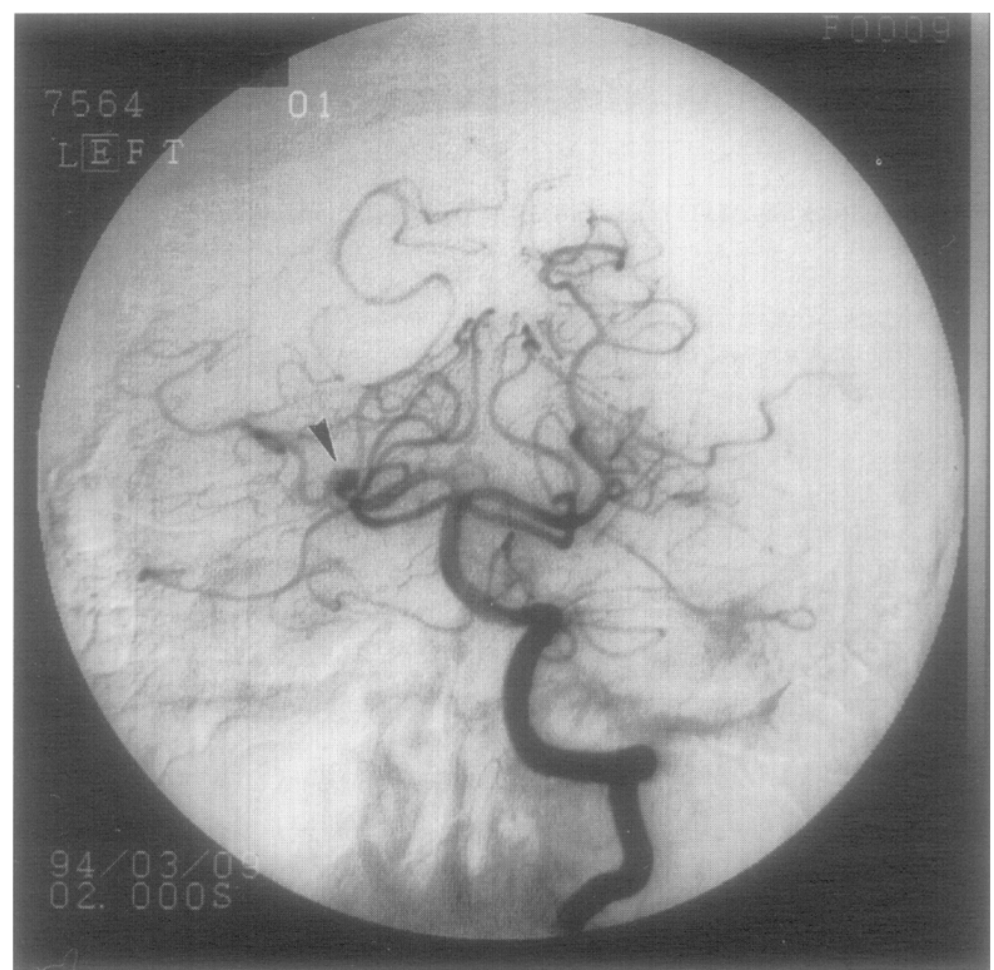

Fig. 1. Vertebral artery angiogram showing $5 \mathrm{~mm}$ aneurysm in the $\mathrm{P} 2$ portion of the right posterior cerebral artery (arrow).

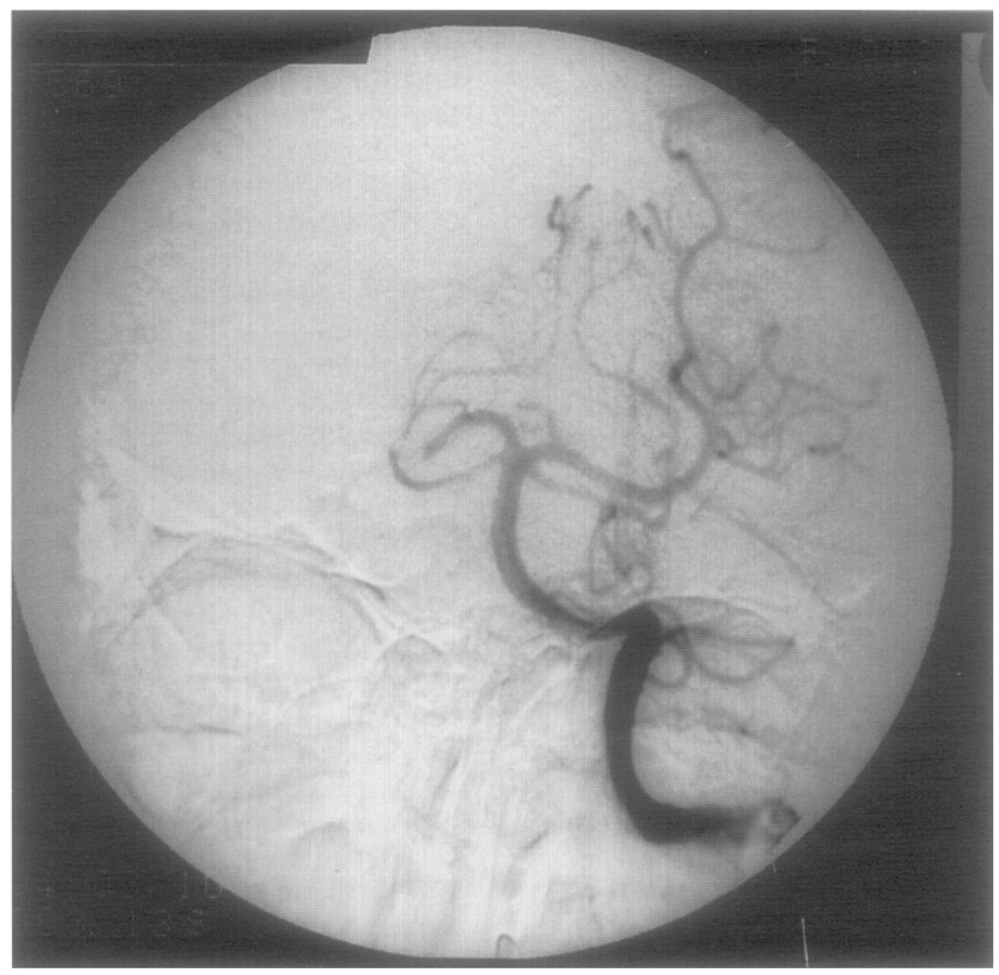

Fig. 2. Vertebral artery angiogram 15 days after endovascular embolization. The aneurysm and the distal portion of the parent artery are not visible. 
approach, and simple observation with high-dose intravenous antibiotics. ${ }^{5}$ In a critical situation such as this case, however, less invasive treatment might be advisable. Urgent cerebral angiography and an endovascular approach to mycotic aneurysms, especially for solitary and peripheral lesions, is an acceptable option for some patients with active endocarditis and severely damaged hearts.

\section{REFERENCES}

1. Bohmfalk GL, Story JL, Weissinger JP, Brown WE. Bacterial intracranial aneurysms. J Neurosurg 1978;48: 369-82.
2. Salgado AV. Central nervous system complications of infective endocarditis. Stroke 1991;22:1461-3.

3. Hart RG, Kagan-Hallet K, Joerns SE. Mechanisms of intracranial hemorrhage in infective endocarditis. Stroke 1987;18:1048-56.

4. Frizzell RT, Vitek JJ, Hill DL, Fisher WS 3rd. Treatment of a bacterial (mycotic) intracranial aneurysm using an endovascular approach. Neurosurgery 1993; 32:852-4.

5. Moskowitz MA, Rosenbaum AE, Tyler HA. Angiographically monitored resolution of cerebral mycotic aneurysms. Neurology 1974;24:1103-8.

\title{
COMBINED CORONARY ARTERY BYPASS GRAFTING AND EXCISION OF ADRENAL PHEOCHROMOCYTOMA
}

\author{
Peng W. Seah, MB, BCh, BAO, Robert Costa, MB, BS, and Hugh Wolfenden, FRACS, \\ Little Bay, New South Wales, Australia
}

The treatment of a patient with an adrenal medullary pheochromocytoma ${ }^{1}$ who also requires surgical revascularization for coronary artery disease presents a rare and interesting challenge. Previously documented experience with this combination of conditions is scarce, and to our knowledge this is the first reported case in which combined coronary artery bypass grafting (CABG) and excision of an adrenal pheochromocytoma was performed.

A 69-year-old woman had a history of an acute anterior myocardial infarction 3 months previously. Coronary angiography revealed severe triple-vessel disease, with an ejection fraction of $49 \%$ and association with anteroapical and inferior hypokinesia. She had New York Heart Association functional class IV angina and was receiving maximal antianginal therapy. Her medical background included a 3-year history of hypertension being treated with metoprolol at $100 \mathrm{mg}$ twice daily and lisinopril at 2.5 mg daily.

While in the hospital with unstable angina and awaiting surgical revascularization, the patient was examined for right upper quadrant abdominal pain and deteriorating liver function. Abdominal ultrasonography and computed tomographic scan (Fig. 1) revealed a well-defined mass measuring approximately $5 \mathrm{~cm}$ in diameter below the right lobe of the liver and arising from the right adrenal gland. Urinary catecholamine levels were elevated, and scintigraphy with metaiodobenzylguanidine labeled with iodine 131 showed this to be a functioning mass consistent with a

From the Department of Cardiothoracic Surgery, The Prince Henry Hospital, Little Bay, New South Wales, Australia.

J Thorac Cardiovasc Surg 1995;110:559-60

Copyright 1995 by Mosby-Year Book, Inc.

$0022-5223 / 95 \$ 3.00+0 \quad \mathbf{1 2 / 5 4 / 6 2 6 1 8}$ pheochromocytoma. An $\alpha$-adrenoceptor blockade was started with oral phenoxybenzamine and the dosage was stabilized at $20 \mathrm{mg}$ three times daily after 3 weeks; this dosage was limited by symptomatic postural hypotension and tachycardia. Oral $\beta$-adrenoceptor blockade was continued with metoprolol.

A combined surgical procedure was performed in cooperation with the urologists. In an effort to reduce perioperative bleeding, a low-dose regimen of aprotinin was given at a dosage of $500,000 \mathrm{U}$ before and during bypass, with a further $200,000 \mathrm{U}$ given after the bypass. The patient underwent cardiopulmonary bypass (CPB). With mild systemic hypothermia and intermittent cold blood cardioplegia, the left internal mammary artery was anastomosed to the left anterior descending artery and saphenous vein grafts were anastomosed to the marginal circumflex and distal right coronary arteries. CPB was then withdrawn with surprising ease. After decannulation, the cannulating tourniquets were snared without being tied to facilitate rapid reintroduction of CPB in case this should be needed to control extremes of blood pressure during removal of the pheochromocytoma. The median sternotomy was extended as a midline laparotomy to just above the umbilicus, splitting the fibrous central part of the diaphragm to just above the inferior vena cava. The left lobe of the liver and the duodenum were mobilized, the perinephric fascia were incised, and the pheochromocytoma was exposed. The drainage veins were clipped and divided, and the tumor was removed. The patient remained in hemodynamically stable condition throughout, with no major swings in blood pressure. The sternotomy and laparotomy wounds were routinely closed, and the patient returned to the recovery room in a stable and satisfactory condition. Throughout the postoperative course, the patient's blood pressure remained remarkably 